EPJ Web of Conferences 52, 01009 (2013)

DOI: $10.1051 /$ epjconf/20135201009

C) Owned by the authors, published by EDP Sciences, 2013

\title{
Results from NA61/SHINE
}

\author{
M. Unger ${ }^{1,}$ a for the NA61/SHINE Collaboration \\ ${ }^{1}$ Institut für Kernphysik, Karlsruher Institut für Technologie, Postfach 3640, D - 76021 Karlsruhe
}

\begin{abstract}
In this paper we summarize recent results from NA61/SHINE relevant for heavy ion physics, neutrino oscillations and the interpretation of air showers induced by ultra-high energy cosmic rays.
\end{abstract}

\section{The NA61/SHINE Experiment}

NA61/SHINE (SHINE = SPS Heavy Ion and Neutrino Experiment) [1] is a multi-purpose fixed target experiment to study hadron production in hadron-nucleus and nucleus-nucleus collisions at the CERN Super Proton Synchrotron (SPS). Among its physics goals are precise hadron production measurements for improving calculations of the neutrino beam flux in the $\mathrm{T} 2 \mathrm{~K}$ neutrino $\mathrm{o}$ cillation experiment [2] as well as for more reliable sim lations of hadronic interactions in air showers. Moreove $p+p, p+\mathrm{Pb}$ and nucleus + nucleus collisions are measure to allow for a study of the properties of the onset of $d$ confinement and a search for the critical point of strong interacting matter (see e.g. Ref. [3]).

The layout of the NA61/SHINE detector is sketch in Fig. 1. A set of scintillation and Cherenkov counters : well as beam position detectors upstream of the spectron eter provide timing reference, identification and positic measurements of the incoming beam particles. Large tim projection-chambers (TPCs) inherited from the NA49 e: periment [4] are used to measure the charge and momes tum of particles. The momentum resolution, $\sigma(1 / p)$ $\sigma(p) / p^{2}$, is about $10^{-4}(\mathrm{GeV} / c)^{-1}$ at full magnetic field ar the tracking efficiency is better than $95 \%$. Particle iden 1 fication is achieved by measuring the energy loss aloriy the tracks in the TPCs and by determining their velocity from the time of flight provided by large scintillator walls placed downstream of the TPCs. The centrality of nucleusnucleus collisions can be estimated using the measurement of the energy of projectile spectators with a calorimeter [5] located behind the time of flight detectors. For nucleonnucleus collisions, the centrality is determined by counting low momentum particles from the target (so called 'gray protons') with a small TPC around the target.

NA61/SHINE started data taking in 2007. After a first run with proton on carbon at $31 \mathrm{GeV} / c$, the data acquisition system was upgraded during 2008 to increase the event recording rate by a factor of $\approx 10$. In the last four years, a wealth of data has been recorded by the exper-

\footnotetext{
a. e-mail: Michael.Unger@kit.edu
}

iment at beam momenta ranging from 13 to $350 \mathrm{GeV} / c$ with various beam particles and targets. In this paper we present results obtained from the data relevant for heavy ion physics, neutrino oscillations and the interpretation of air showers at ultra-high energies.

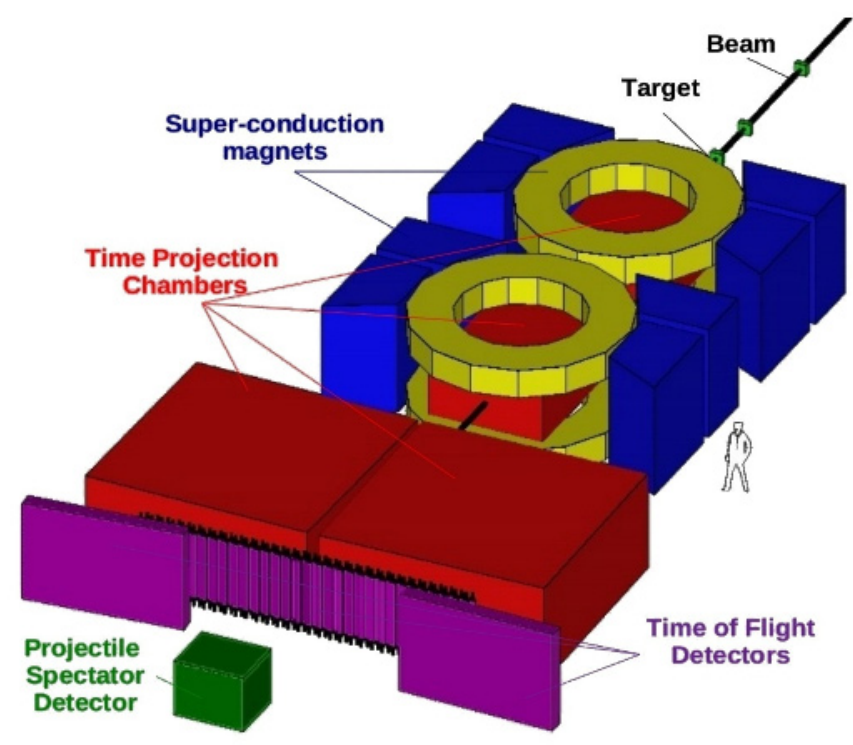

Figure 1. Schematic layout of the NA61/SHINE experiment.

\section{Measurements of $p+\mathbf{C}$ Interactions for the Improvement of Neutrino Flux Calculations}

Measurements of the particle emission from targets used to create neutrino beams are important for a precise interpretation of long-baseline neutrino oscillation experiments such as Tokai-to-Kamioka (T2K) [2]. Two types of measurements have been performed by NA61/SHINE to aid the T2K calculations of the neutrino fluxes: interactions of proton on a replica of the T2K target (a $90 \mathrm{~cm}$ long 


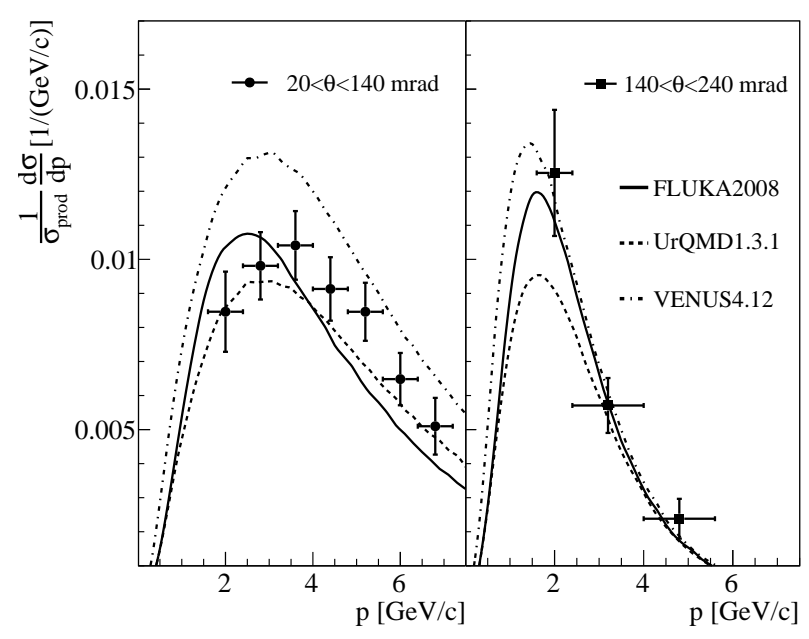

Figure 2. Comparison of measured $K^{+}$spectra in $p+\mathrm{C}$ interactions at $31 \mathrm{GeV} / c$ with model predictions. The vertical error bars on the data points show the total (stat. and syst.) uncertainty. The lines indicate predictions using Venus [9], Fluka [10] and UrQMD [11, 12].

graphite rod) and thin $(2 \mathrm{~cm})$ target measurements to allow the measurement of single proton-carbon interactions. Both measurements were performed with a $31 \mathrm{GeV} / c$ proton beam, similar to the one provided at J-PARC. A total of $0.2 \times 10^{6}$ events were recorded during the data taking in 2007 and more statistics were collected in $2009\left(4 \times 10^{6}\right.$ events) and $2010\left(10 \times 10^{6}\right.$ events $)$. For the first time, the kinematic phase space of pions and kaons exiting the target and producing neutrinos in the direction of the near and far detectors of a long-baseline neutrino oscillation experiment is fully covered by a single hadron production experiment.

First results on pion and kaon yields have already been published [6,7] and used in the T2K data analysis [8]. An example of a recent particle yield measurement for $\mathrm{T} 2 \mathrm{~K}$ is the inclusive yield of positive kaons in $\mathrm{p}+\mathrm{C}$ interactions at $31 \mathrm{GeV} / c$ which is shown Fig. 2. The knowledge of charged kaon yields is important for T2K because kaons generate the high energy tail of the neutrino beam and contribute substantially to the intrinsic $v_{e}$ component. As can be seen, none of the superimposed model predictions can fully describe the small-angle data from 20 to 140 mrad. Because of these shortcomings of hadronic interaction models and similar deficits in case of a comparison of predicted pion yields to NA61/SHINE data (cf. [6]), neutrino flux predictions cannot be used directly to interpret oscillation data, but need to be modified to match the NA61/SHINE measurements. For this purpose flux simulations are re-weighted to match the measured secondary particle yields, either on an interaction-by-interaction basis using the thin-target data, or at the surface of the T2K target by using data collected with the replica-target [13]. As can be seen in Fig. 3, the resulting calculated neutrino spectra at the $\mathrm{T} 2 \mathrm{~K}$ far detector are in excellent agreement using either of these two methods.

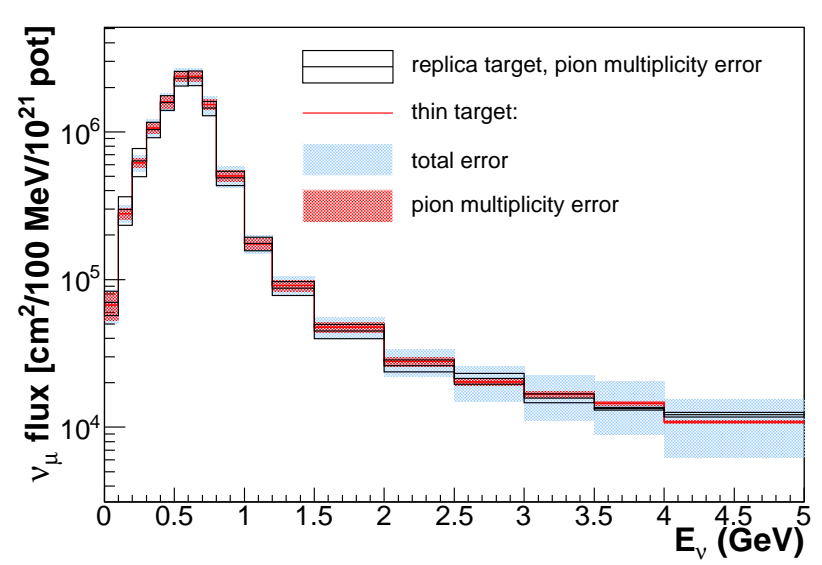

Figure 3. Re-weighted $v_{\mu}$ flux predictions at the far detector of T2K based on the NA61/SHINE thin-target and replica-target data.

\section{NA61/SHINE Results for the Interpretation of Cosmic Ray Air Showers}

Cosmic rays initiate extensive air showers (EAS) when they collide with the nuclei of the atmosphere. The interpretation of EAS data as for instance recorded by the Pierre Auger Observatory [14], KASCADE [15] or IceTop [16] relies to a large extent on the understanding of these air showers and specifically on the correct modeling of hadron+air interactions that occur during the shower development. The relevant particle energies span a wide range from primary energies of $\gtrsim 10^{20} \mathrm{eV}$ down to energies of $10^{9} \mathrm{eV}$. The mesons that decay to muons at ground level typically originate from low energy interactions in the late stages of an air shower. Depending on the primary energy and detection distance, the corresponding interaction energies are between 10 and $1000 \mathrm{GeV}$ and the modeling of the corresponding low energy interactions contribute at least $10 \%$ to the overall uncertainty of the predicted muon number at ground (see e.g. Refs. [17-20]).

Unfortunately, there exist no comprehensive and precise particle production measurements for the most numerous projectile in air showers, the $\pi$-meson. Therefore, new data with pion beams at 158 and $350 \mathrm{GeV} / c$ on a thin carbon target (as a proxy for nitrogen) were collected by the NA61/SHINE experiment at the CERN SPS and preliminary results from this data set were presented at this conference for the first time.

The production cross section in $\pi^{-}+\mathrm{C}$ interactions was determined in a similar manner as described in Ref. [6], by correcting the experimental interaction cross section by residual contributions from elastic and quasi-elastic scattering as well as for the inelastic contribution to which the NA61/SHINE interaction trigger is not sensitive. The uncertainties of the measurement is currently dominated by the model-dependence of this correction. Preliminary val- 


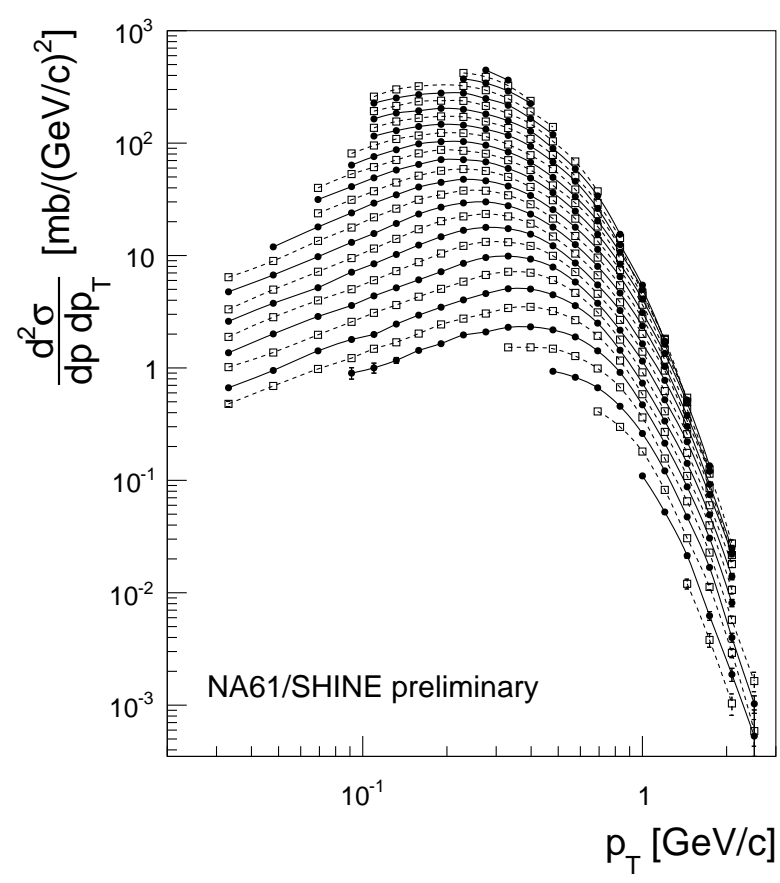

(a) $h^{-}$at $158 \mathrm{GeV} / c$

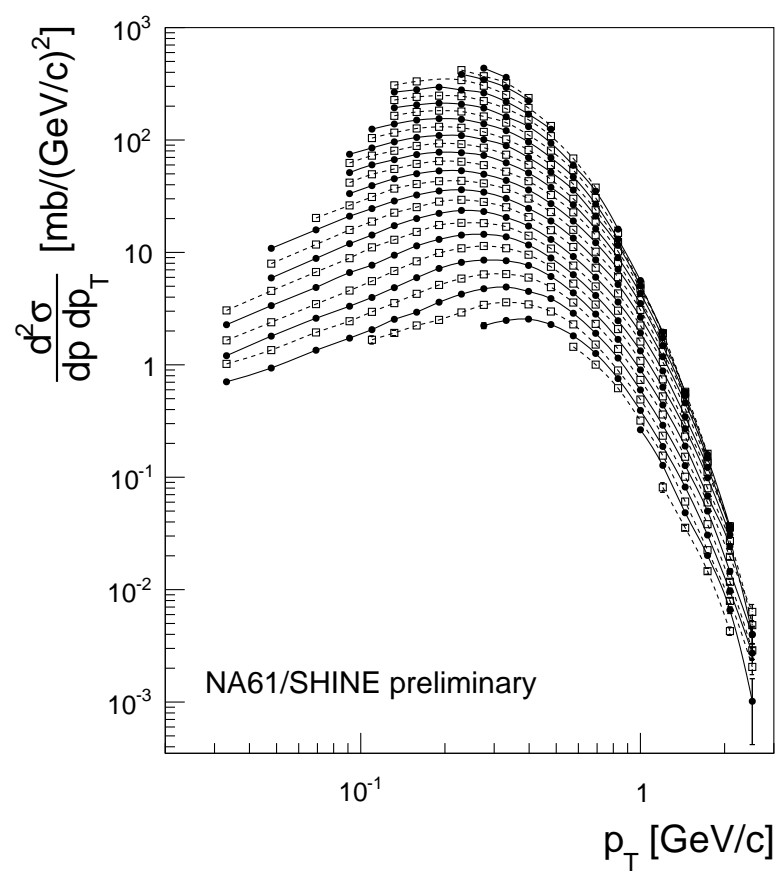

(c) $h^{-}$at $350 \mathrm{GeV} / c$

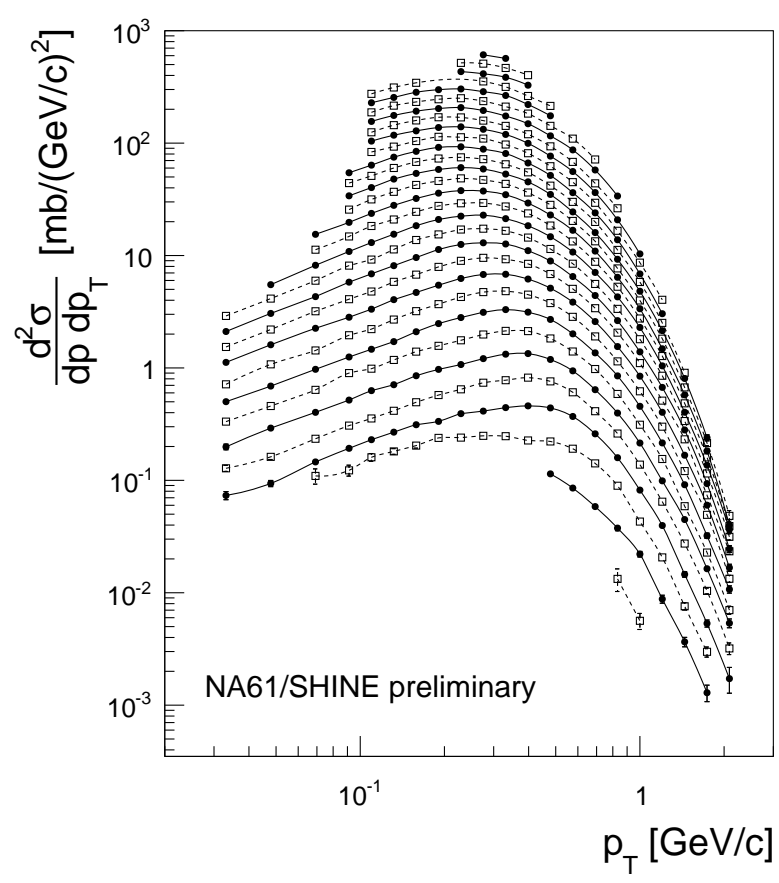

(b) $h^{+}$at $158 \mathrm{GeV} / c$

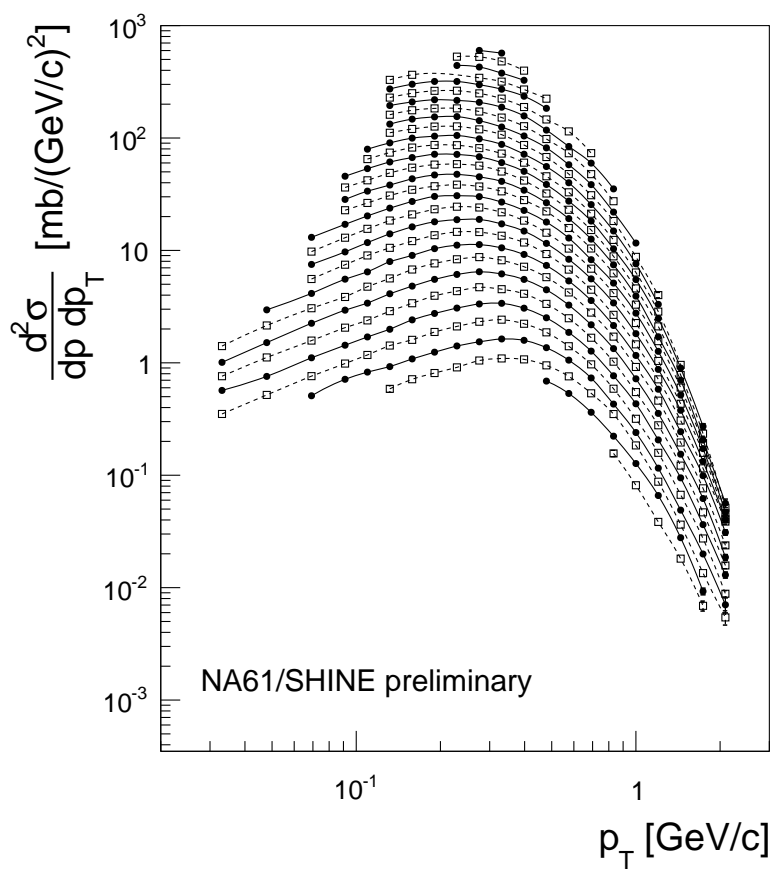

(d) $h^{+}$at $350 \mathrm{GeV} / c$

Figure 4. Inclusive $p_{\mathrm{T}}$-spectra of charged hadrons produced in $\pi^{-}+\mathrm{C}$ interactions at 158 and $350 \mathrm{GeV} / c$. In each figure, the particle momentum $p$ ranges from 0.6 to $121 \mathrm{GeV} / c$ in steps of $\log (p /(\mathrm{GeV} / c))=0.08$ from top to bottom.

ues are $\sigma_{\text {prod }}=172 \pm 2$ (stat.) \pm 4 (syst.) and $178 \pm 2$ (stat.) \pm 4 (syst.) at 158 and $350 \mathrm{GeV} / c$ respectively [22]. This measurement is compatible with previous results $[27,28]$ and already gives the most precise value of the production cross section at around $160 \mathrm{GeV} / c$.

The momentum spectra of charged hadrons in $\pi^{-}+\mathrm{C}$ interactions at 158 and $350 \mathrm{GeV} / c$ are presented in Fig. 4.
These spectra were obtained within a fiducial phase space in the NA61 detector, for which the detection and selection efficiency for charged tracks is close to unity, and corrected for feed-down and track loss using the average correction predicted by the Venus and EPos event generators after simulation of the detector response [23]. The trigger bias is corrected for by studying the track loss in a sub-sample 

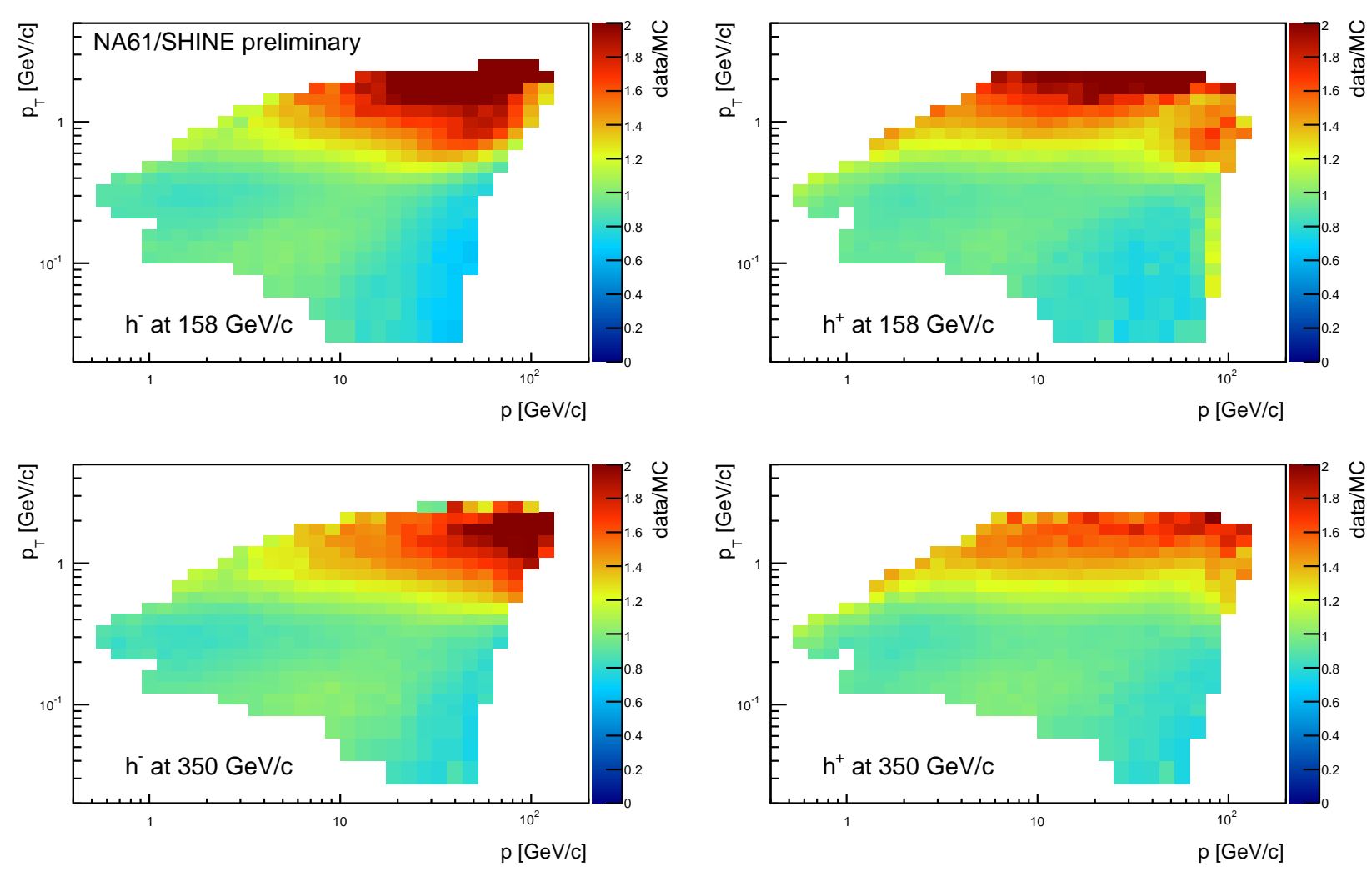

Figure 5. Comparison of measured charged hadron production yields in $\pi^{-}+\mathrm{C}$ interactions at 158 and $350 \mathrm{GeV} / c$ to predictions from Epos 1.99. Colors denote the ratio of data over MC and the different panels are for different charges and beam energies as indicated by the labels. Note that the color scale is limited, i.e. the maximum value to be understood as data/MC $\geq 2$.

of unbiased beam-trigger data. Only phase-space regions for which the overall model correction is below $20 \%$ and for which the total systematic uncertainty is smaller than $20 \%$ are displayed in Fig. 4. The uncertainties shown are the total uncertainties including the statistical uncertainty

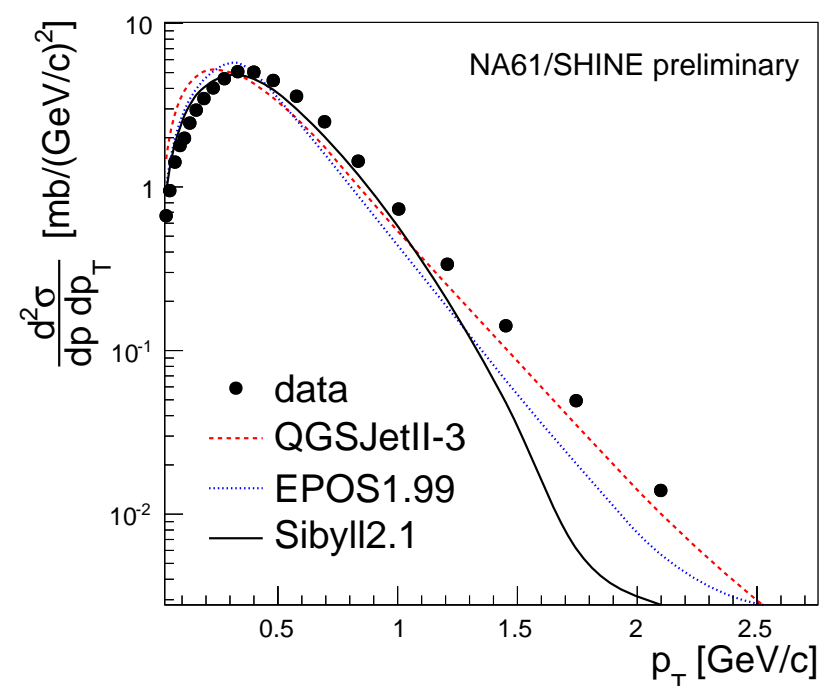

Figure 6. Transverse momentum spectrum of negatively charged hadrons produced in $\pi^{-}+\mathrm{C}$ interactions at $158 \mathrm{GeV} / c$ beam momentum at $\langle p\rangle=10.4 \mathrm{GeV} / c$. and systematics from the model correction, normalization, trigger bias, calibration and track topology.

These preliminary measurements are already useful to judge the quality of event generators used in air-shower simulations. An example of the $p_{\mathrm{T}}$ distribution of negatively charged hadrons produced in $\pi^{-}+\mathrm{C}$ interactions at a beam momentum of $158 \mathrm{GeV} / c$ is shown in Fig. 6 for particle momenta with $\langle p\rangle=10.4 \mathrm{GeV} / c$ and compared to predictions by QGSJetII-03 [26], Sibyll2.1 [25] and Epos1.99 [24]. As can be seen, none of these hadronic interaction models which are used to simulate air showers can reproduce that data and especially SIBYLL2.1 predicts a much too steep spectrum at high transverse momenta.

The full data set is compared to the predictions of the Epos1.99 model in Fig. 5 where the ratio of data over $\mathrm{MC}$ is shown. It can be seen that the underestimation of charged hadron production at large transverse momenta, which was illustrated in Fig. 6 at one particular momentum, is present at all momenta (the same holds true for SibYLL). Of all the models studied QGSJetII-03 describes our data best with only a small deficit of tracks with high $p_{\mathrm{T}}$ at large particle momentum but slightly too many particles at low traverse momenta.

It is planned to study these shortcomings of the models in more detail by measuring the spectra of identified hadrons. Moreover, NA61/SHINE will be able to validate the measurement of proton and anti-proton production in 

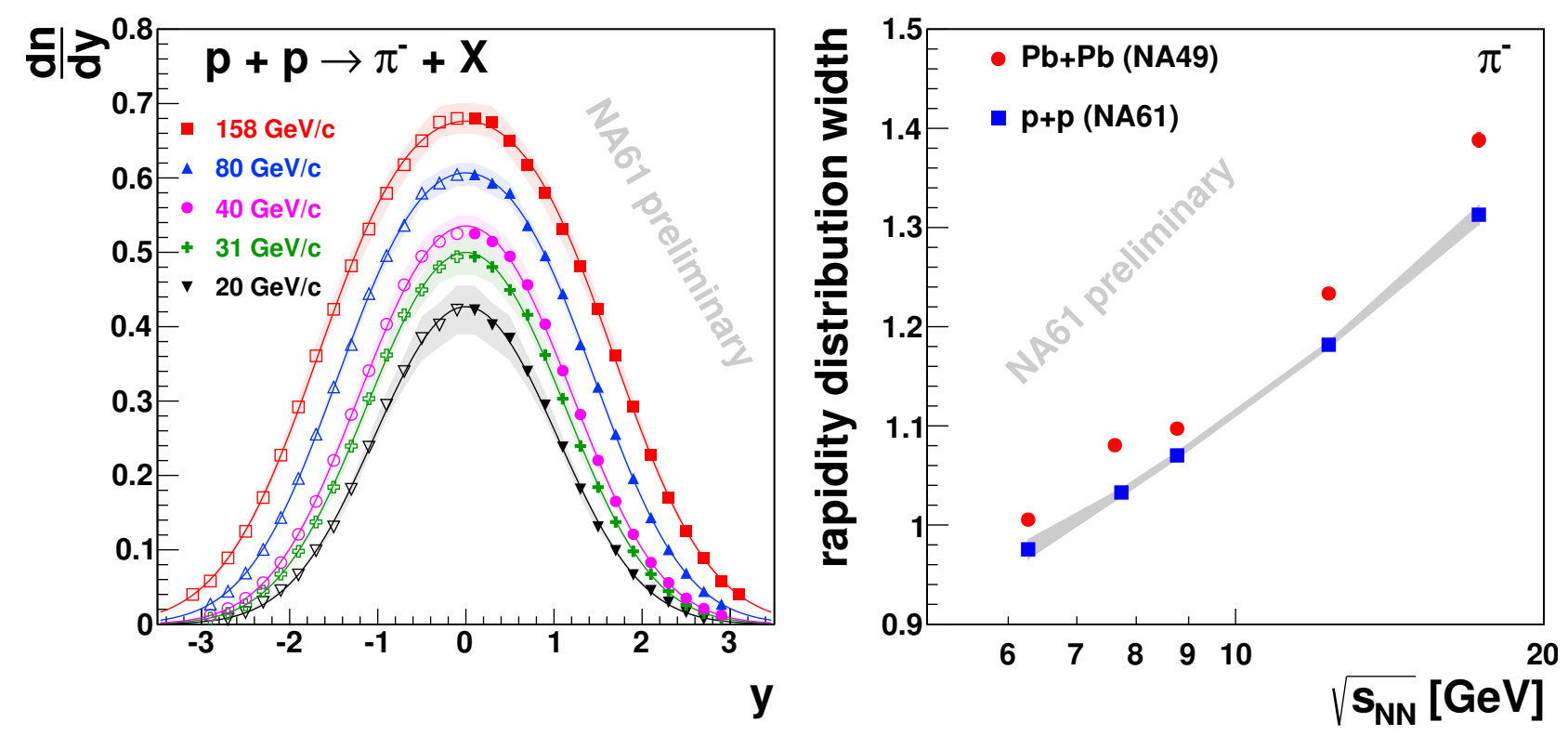

Figure 7. Left: $m_{\mathrm{T}}$-integrated $\pi^{-}$rapidity spectra in $p+p$ interactions at $20-158 \mathrm{GeV} / c$ obtained with the $h^{-}$method and fitted with a sum of two symmetrically displaced Gaussian functions. Full symbols represent the preliminary measurement and open symbols are the same data reflected around zero rapidity. Right: $\pi^{-}$width (RMS) of the rapidity spectrum as a function of energy, compared with $\mathrm{Pb}+\mathrm{Pb}$ data $[31,32]$.

$\pi+\mathrm{C}$ interactions from Ref. [29] on which mostly the enhanced baryon production is based on that has been proposed in Ref. [24] as a possibility to enlarge the number of muons in air showers. In addition, the NA61/SHINE data set offers the possibility to constrain the $\rho^{0}$ production in $\pi+\mathrm{C}$ which may be equally important for muons observed in air showers as the baryon fraction (see e.g. Ref. [30]).

\section{The NA61/SHINE Heavy lon Program}

Within its heavy ion program, NA61/SHINE aims to discover the critical point of strongly interacting matter as well as to establish the properties of the onset of deconfinement (see Ref. [3] and references therein). The full experimental program is illustrated in Fig. 8 and consists of a detailed scan of various system sizes and interaction energies.

As a first step, $p+p$ interactions were measured at six energies in 2009-2011 to serve as a reference data set for the subsequent measurement of light and medium size ion reactions in the range of $\sqrt{s_{N N}}=5-20 \mathrm{GeV}$. In this conference we presented preliminary spectra of $\pi^{-}$in $p+p$ collisions at $20,31,40,80$, and $158 \mathrm{GeV} / c$ that were obtained using the so-called $h^{-}$analysis. The analysis is based on the fact that the majority of produced negatively charged particles are pions. Contribution of other particles (mostly $\mathrm{K}^{-}$and feed-down) is corrected for using Monte-Carlo simulations. The corresponding correction is calculated as arithmetic average of VENUs [9] and Epos [24] corrections and the difference between them contributes to the systematic error. The detector effects (acceptance, inefficiency) are corrected for using Monte Carlo as well. This approach allows obtaining $\pi^{-}$spectra in full measured phase space in a uniform way. Non-target interactions, i.e. collisions with air and the detector material, are subtracted using events measured with the empty liquid-hydrogen target. The transverse-mass spectra were found to follow an exponential distribution with $m_{T}$. Therefore, they can be extrapolated to full phase space using an exponential fit to the high $m_{T}$ tail of the spectra to obtain the $m_{T}$-integrated rapidity spectra presented in Fig. 7 (left). The spectra are well described by a sum of two symmetrically displaced Gaussian functions. The widths of the rapidity spectra are presented in Fig. 7 (right) and compared to $\mathrm{Pb}+\mathrm{Pb}$ data.

In addition to these results, preliminary inclusive spectra of identified pions, protons and kaons are also available from NA61/SHINE that were obtained by using the energy deposit in the TPCs for particle identification. With the help of the 'identity'-method described in Ref. [33], estimates of multiplicity fluctuations in $p+p$ interactions could be given. These measurements will form the reference data set for the full ion program. As can be seen in Fig. 8, NA61/SHINE already finished the energy scan with light Be ions that were obtained by fragmenting primary $\mathrm{Pb}$ ions from the SPS [34]. Data taking will resume with $p+\mathrm{Pb}$ interactions in 2014 and the remaining two system sizes, $\mathrm{Ar}+\mathrm{Ca}$ and $\mathrm{Xe}+\mathrm{La}$, will be measured in 2015 and 2016. Within a possible extension of the approved physics 


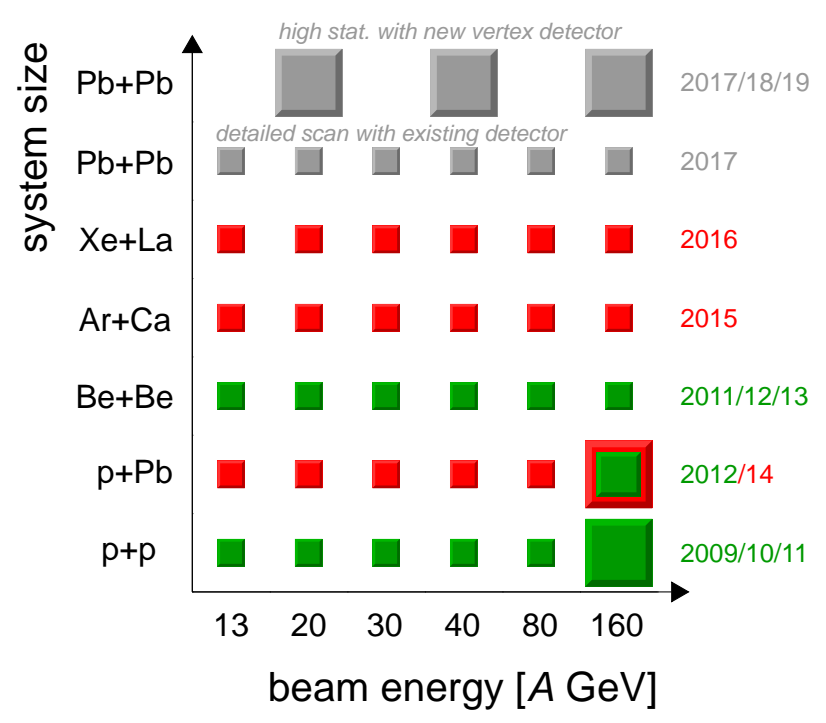

Figure 8. The NA61/SHINE data taking schedule for the ion program and its proposed extension for the period 2017-2019. Big boxes denote large ( $~\left(5 \times 10^{7}\right.$ events) and small boxes medium size $\left(\sim 1-5 \times 10^{6}\right.$ events) data sets. Green boxes are recorded data, red boxes denote runs to be taken within the approved physics program and gray boxes denote the proposed extension.

program, it is foreseen to provide more $\mathrm{Pb}+\mathrm{Pb}$ data at six energies and to study $D$-meson production in heavy ion collisions at SPS energies with high statistics runs and a new vertex detector [35].

\section{Conclusions}

In this article, we summarized results from the multipurpose experiment NA61/SHINE at the CERN SPS. In the light of the main focus of this conference, Very High Energy Cosmic Ray Interactions, it is worth noting that although the different measurements are motivated by different physics goals, all the hadron-nucleus and protonproton data collected by NA61/SHINE are valuable for the tuning of hadronic interaction models for the understanding of air showers. The published NA61/SHINE data on $p+\mathrm{C}$ interactions at $31 \mathrm{GeV} / c$ [6] have already been used to fix [11] the Uromd1.3.1 model [12] and to further strengthen [37] the case against the use of GHEIsHa [36]. Both of these models are available in CoRsIKA [38] to simulate low energy interactions in air shower. Moreover, recent comparisons [39] of NA61/SHINE data to Epos suggest that high-energy interaction models need further tuning even for 'well-known' reactions like $p+p$.

Together with the special cosmic ray runs for pioncarbon interactions, the rich data set of NA61/SHINE will thus be very useful to reduce uncertainties in air shower calculations and increase the knowledge on interactions in the late stages of the shower development below a TeV.

\section{References}

[1] N. Antoniou et al. [NA61/SHINE Collaboration], CERN-SPSC-2007-004, (2007); CERN-SPSC-2007019, (2007); N. Abgrall et al. [NA61/SHINE Collaboration], CERN-SPSC-2008-018, (2008).

[2] K. Abe et al. [T2K Collaboration], Nucl. Instrum. Meth. A 659 (2011) 106.

[3] M. Gazdzicki, M. Gorenstein, P. Seyboth, Acta Phys. Polon. B42 (2011) 307.

[4] S. Afanasev et al. [NA49 Collaboration], Nucl. Instrum. Meth. A 430 (1999) 210.

[5] A. Ivashkin et al., arXiv:1205.4864.

[6] N. Abgrall et al. [NA61/SHINE Collaboration], Phys. Rev. C 84 (2011) 034604.

[7] N. Abgrall et al. [NA61/SHINE Collaboration], Phys. Rev. C 85 (2012) 035210.

[8] K. Abe et al. [T2K Collaboration], Phys. Rev. Lett. 107 (2011) 041801; Phys. Rev. D 87 (2013) 012001.

[9] K. Werner, Nucl. Phys. A525, 501c (1991); Phys. Rep. 232, 87 (1993).

[10] A. Fasso et al., CERN-2005-10 (2005); G. Battistoni et al., AIP Conf. Proc. 896 (2007) 31.

[11] V. Uzhinsky, arXiv:1107.0374 [hep-ph].

[12] S.M. Bass et al., Prog. Part. Nucl. Phys. 41225 (1998); M. Bleicher et al., J. Phys. G: Nucl. Part. Phys. 25, 1859 (1999).

[13] N. Abgrall et al. [NA61/SHINE Collaboration], Nucl. Instrum. Meth. A 701 (2013) 99.

[14] J. Abraham et al. [Pierre Auger Collaboration], Nucl. Instrum. Meth. A 523 (2004) 50.

[15] T. Antoni et al. [KASCADE Collaboration], Nucl. Instrum. Meth. A 513 (2003) 490.

[16] R. Abbasi et al. [IceCube Collaboration], Nucl. Instrum. Meth. A 700 (2013) 188.

[17] D. Heck et al., Proc. 28th ICRC, (2003) 279.

[18] H.-J. Drescher et al., Astropart. Phys. 21 (2004) 87.

[19] C. Meurer et al., Czech. J. Phys. 56 (2006) A211.

[20] I. C. Mariş et al., Nucl. Phys. Proc. Suppl. 196 (2009) 86.

[21] I. C. Mariş et al. [NA61/SHINE Collaboration], Proc. 31st ICRC, (2009).

[22] M. Haug, Messung des Wirkungsquerschnitts von Pion-Kohlenstoff-Wechselwirkungen mit Hilfe des NA61 Detektors, Diploma Thesis, KIT, 2012.

[23] M. Ruprecht, Measurement of the Spectrum of Charged Hadrons in $\pi^{-}+C$ Interactions with the NA61 Experiment, Diploma Thesis, KIT, 2012.

[24] T. Pierog and K. Werner, Phys. Rev. Lett. 101 (2008), 171101.

[25] E.J. Ahn et al., Phys. Rev. D80 (2009) 094003.

[26] S.S. Ostapchenko, Nucl. Phys. Proc. Suppl. 151 (2006), 143;

[27] A. Schiz et al., Phys. Rev. D 21 (1980) 3010.

[28] U. Dersch et al. [SELEX Collaboration], Nucl. Phys. B 579 (2000) 277.

[29] D. S. Barton et al., Phys. Rev. D 27 (1983) 2580. 
[30] H. -J. Drescher, Phys. Rev. D 77 (2008) 056003.

[31] S. V. Afanasiev et al. [NA49 Collaboration], Phys. Rev. C 66 (2002) 054902.

[32] C. Alt et al. [NA49 Collaboration], Phys. Rev. C 77 (2008) 024903.

[33] M. Gazdzicki, K. Grebieszkow, M. Mackowiak and S. Mrowczynski, Phys. Rev. C 83 (2011) 054907

[34] H. Ströbele and I. Efthymiopoulos, CERN Cour. 52N4 (2012) 33.
[35] N. Abgrall et al. [NA61/SHINE Collaboration], NA61/SHINE plans beyond the approved program, CERN-SPSC-2012-022; SPSC-P-330-ADD-6.

[36] H. Fesefeldt, Aachen Report No. PITHA-85-02 (1985)

[37] M. Unger for the NA61/SHINE Collaboration, Proc. 35th ICHEP (2010), arXiv:1012.2604.

[38] D. Heck et al., FZK Report No. FZKA-6019, 1998.

[39] S. Pulawski for the NA61/SHINE Collaboration, talk at the 8th CPOD (2013). 